\title{
Co-occurrence of two cases of progressive multifocal leukoencephalopathy in a natalizumab "infusion group"
}

Multiple Sclerosis Journal

19(9) $1213-1215$

(C) The Author(s) 2012

Reprints and permissions:

sagepub.co.uk/journalsPermissions.nav DOI: | 0.1 | 77/| 3524585|2466|65 msj.sagepub.com

(S)AGE

\author{
J Havla', A Berthele'2, T Kümpfel', M Krumbholz', A Jochim², \\ H Kronsbein ${ }^{2}$, C Ryschkewitsch ${ }^{4}$, P Jensen ${ }^{4}$, K Lippmann ${ }^{3}$, \\ B Hemmer ${ }^{2}$, E Major ${ }^{4}$ and R Hohlfeld '
}

\begin{abstract}
We observed two cases of progressive multifocal leukoencephalopathy (PML) that occurred in the same "infusion group". The group consisted of four patients with relapsing-remitting multiple sclerosis (RRMS) who had been treated with natalizumab (NAT) in the same medical practice for more than four years at the same times and in the same room, raising concerns about viral transmission between members of the infusion group.

DNA amplification and sequence comparison of the non-coding control region (NCCR) of JC virus (JCV) present in cerebrospinal fluid (CSF) samples from PML patients \#I and \#2 revealed that the amplified JCV sequences differed from the JCV archetype. The NCRR of the viral DNA was unique to each patient, arguing against the possibility of viral transmission between patients. Statistical considerations predict that similar co-occurrences of PML are likely to happen in the future.
\end{abstract}

\section{Keywords}

Natalizumab, multiple sclerosis, progressive multifocal leukoencephalopathy, PML, JC virus

Date received: 9th August 2012; revised: II th September 2012; accepted: 4th October 2012

\section{Introduction}

Progressive multifocal leukoencephalopathy (PML) is an opportunistic infection caused by JC virus (JCV). PML is a known risk associated with natalizumab (NAT, Tysabri ${ }^{\circledR}$, Biogen Idec, USA), an anti-alpha-4 integrin monoclonal antibody approved for the treatment of severe and otherwise treatment-resistant relapsing-remitting multiple sclerosis (RRMS). The overall risk of NAT-associated PML, estimated around $0.2 \%$, increases with the number of infusions, positive JCV antibody status, and previous treatment with immunosuppressive agents. ${ }^{1}$ Here we report on two multiple sclerosis (MS) patients who were treated in the same medical practice, and who were diagnosed with PML within four months of each other. They both belonged to an otherwise unrelated "infusion group" of four patients who, for at least four years, received their monthly infusions at the same times and in the same room. They had not previously been treated with an immunosuppressive agent. The coincidental occurrence of two PML cases in the same infusion group has not been described so far. Understandably, the patients and their treating neurologist were concerned about the possibility of viral transmission.

\section{Case reports}

Patient \#1, a 53-year-old male from Germany, received NAT for RRMS from February 2007 through February 2011 (52 infusions). Beforehand, he had failed on beta-interferon therapy, but had never received immunosuppressants. NAT was discontinued because of the diagnosis of PML (March 2011) with cognitive decline and personality change, typical magnetic resonance imaging (MRI) lesions, and high JCV DNA loads $\left(1.7 \times 10^{6} \mathrm{Geq} / \mathrm{ml}\right.$ in cerebrospinal fluid (CSF), $2.4 \times 10^{6} \mathrm{Geq} / \mathrm{ml}$ in serum, tested in Munich, Germany). His neurological condition stabilized after plasmapheresis and

\footnotetext{
'Institute of Clinical Neuroimmunology, Ludwig-Maximilians-University, Germany.

${ }^{2}$ Department of Neurology, Technische Universität München, Germany. 3Practice of Dr Bergmann/Dr Lippmann, Germany.

${ }^{4}$ Laboratory of Molecular Medicine, National Institutes of Health, USA.

Corresponding author:

Reinhard Hohlfeld, Institute of Clinical Neuroimmunology, Klinikum der LMU München, Campus Grosshadern, Marchioninistr. I5, D-8I 377 München, Germany.

Email: reinhard.hohlfeld@med.uni-muenchen.de
} 
prolonged treatment with steroids without signs of immune reconstitution syndrome (IRIS). After six months, he had gradually improved but severe neuropsychological deficits remained (Expanded Disability Status Scale (EDSS) 5.0).

Patient \#2 is a 45-year-old German woman who was started on NAT in November 2007 because of ongoing disease activity despite interferon therapy. She had never been treated with immunosuppressants. During NAT therapy she showed neither clinical nor MRI evidence of disease activity. After patient \#1 was diagnosed with PML, and after she tested positive for anti-JCV-antibodies (Abs), she decided to stop NAT after 49 infusions in July 2011 because of fear of PML. Six weeks later she developed new brainstem symptoms and showed a progressive brainstem-midbrain lesion on MRI. PML was diagnosed in September 2011 after detection of JCV DNA $(31 \mathrm{c} / \mathrm{ml}$ in CSF, $5 \mathrm{c} / \mathrm{ml}$ in serum, Dusseldorf laboratory; EDSS 7.0). She developed severe IRIS, and subsequently stabilized after treatment with steroids and intravenous immunoglobulin. ${ }^{2}$ After six months, she had slightly improved but severe ataxia and ocular motor deficits remained (EDSS 6.0).

After PML was diagnosed in patient \#1, serum JCV Abs were tested in all four patients of the infusion group. Three patients, including patients $\# 1$ and $\# 2$, were JCV Abspositive, one patient negative. After patient \#2 was diagnosed with PML, the two remaining patients, including the JCV Abs-negative patient, decided to stop NAT because they were concerned about infection.

\section{Results}

\section{JCV sequence analysis}

DNA of the JCV non-coding regulatory region (NCRR) was amplified by PCR from the CSF of patients \#1 and \#2 according to published methods. ${ }^{3,4}$ The figure shows comparative alignments of the JCV NCRR sequences from patients \#1 and \#2, as well as representative, hypervariable sequence types IIS, IS, IR and IIR (scaled linear depiction). ${ }^{3}$ The NCRRs from patients $\# 1$ and $\# 2$ are unique. They differ from each other and from the archetype, show deletion of the $\mathrm{D}$ region, and contain repeats typical of JCV variants found in PML tissues. Together, these features strongly argue against the possibility that viral transmission occurred between PML patients \#1 and \#2.

\section{Discussion}

We investigated the possibility of viral transmission between members of the infusion group. It is thought that the circulating genotype or "strain" of JCV is the "archetype" that has a signature nucleotide sequence in its NCRR. It can multiply in the kidney and be excreted into the urine. It also has the ability to enter the bloodstream and to distribute into other tissues. It is thought that in lymphoid tissues a 'rearrangement' takes place in the NCCR that might be specific to each patient. If a virus with an NCCR that has rearranged enters the brain, it can infect oligodendrocytes and initiate PML. The variant JCV can be detected in the CSF by PCR and is similar to what has been identified in the brain of the same patient. The observation that the NCRR regions of the mutated viruses of the two PML patients were different from each other likely excludes the possibility that the JCV variant from one patient spread to the other patient (Figure 1). There remains the remote possibility that an archetype-like strain of JCV spread by de novo infection from one patient to the other at an earlier time-point, and subsequently mutated independently in the two patients. To investigate the possibility of de novo infection, we searched to obtain serum samples taken before NAT was started. We were able to locate a pre-treatment serum sample from each of the two patients who later developed PML (patient \#1 and \#2). Both sera were retrospectively found to be positive for anti-JCV antibodies, speaking against the contingency of de novo infection of these patients.

The occurrence of two cases of PML among four members of an infusion group who had been treated together for more than four years at the same times in the same room of the same medical practice created much concern among the patients and treating neurologist. The probability that two cases of PML occur in a given infusion group is very low. Assuming an overall PML risk of approximately two of 1000, the probability of at least two cases of PML occurring in a given group of four is only $2.4 \times 10^{-5}$. In contrast, the probability of co-occurrence of at least two PML cases taking place anywhere in the world is much higher, as indicated by the following rough estimate. Assuming that all patients are currently treated with NAT in groups of four (100,000 patients treated in 25,000 groups of four), and assuming an individual PML risk of $0.2 \%$, the probability of at least one "PML cooccurrence" worldwide is $45 \%$. Based on these estimates, one would predict that additional PML co-occurrences will happen over time. Furthermore, we roughly estimated the probability that at least two cases of PML might occur in a large neurological practice. Assuming that a neurologist treats 200 MS patients with NAT, $50 \%$ of whom are JCV negative and $50 \%$ seropositive but without prior immunosuppressive treatment, the probability of two or more PML cases among these patients within two to four years of therapy is $8 \%$, based on the figures published by Biogen Idec in July $2012\left(p_{\mathrm{JCV} \text { neg }}=\right.$ $0.09 / 1000 ; p_{\mathrm{JCV} \text { pos/IS neg }}=4.6 / 1000$; calculations in $R$ using binomial distributions). This is a lower limit considering that in reality some of the patients likely were pretreated with an immunosuppressant agent.

Summarizing for our cases, the results of the virological investigations likely excluded the possibility of viral transmission among members of the infusion group. Consequently, there is presently no need to alter current infusion practices, which will be reassuring to patients and neurologists. 


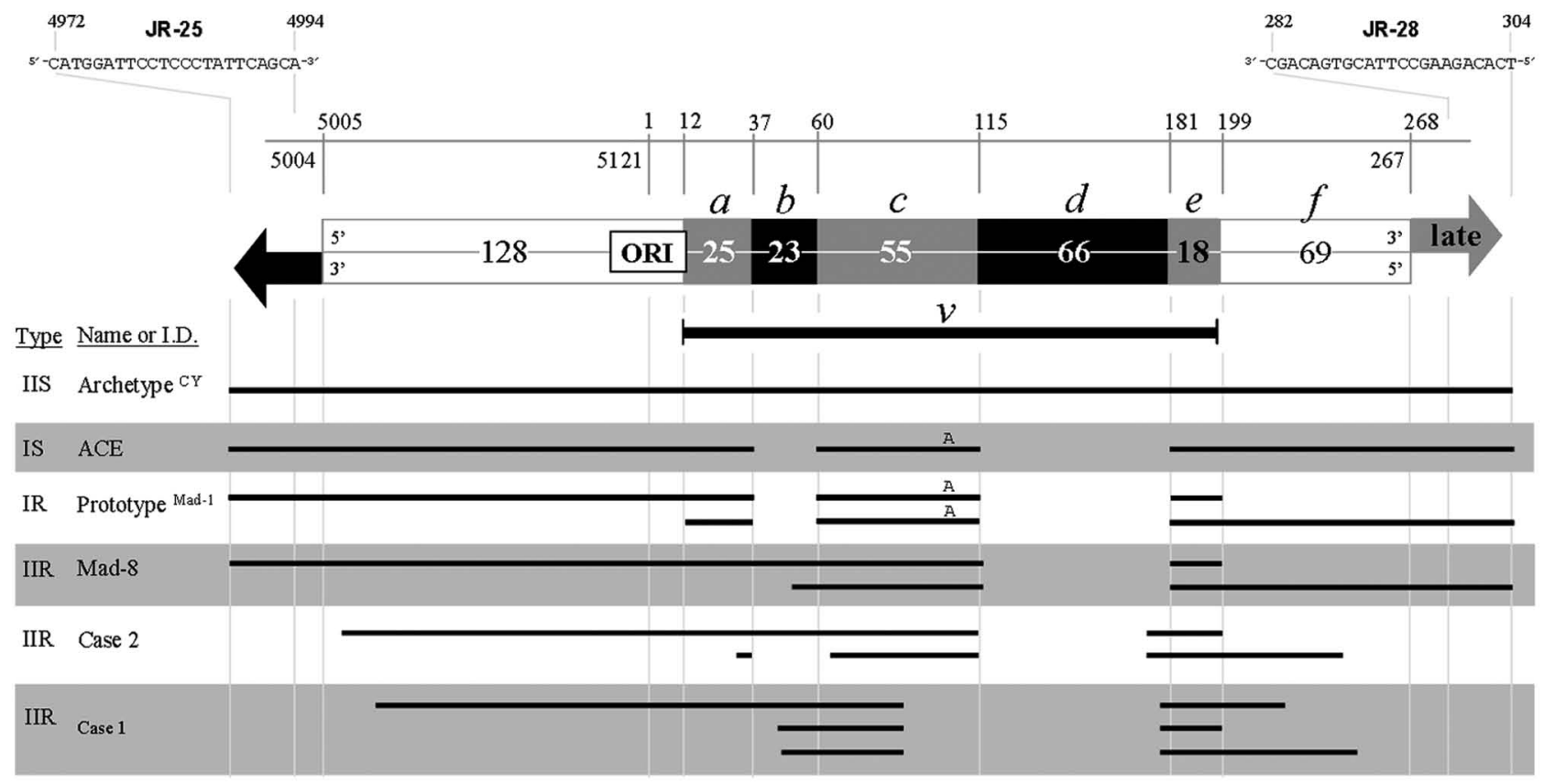

Figure I. Scaled linear depiction of JC virus $(\mathrm{JCV})$ non-coding regulatory region (NCRR) sequences with comparative alignments of representative, hypervariable sequence types (IIS, IS, IR, and IIR ${ }^{3}$ ) and nucleotide sequences from Cases I and 2.

Modified nucleotide-numbering system adapted from the Prototype ${ }^{\text {Mad-I }}$ JCV genome. ${ }^{4}$ The NCRR is positioned between the early (dark arrow on left) and late (light arrow on right) protein coding sequences within the circular, supercoiled, double stranded JCV DNA genome. From the early side, the initial sequence section contains the origin of DNA replication (ORI) followed by sequence sections designated a, b, c, d, e and f. The basepair length of each sequence section is noted. From variant to variant, the dark bar, $v$, denotes sequence sections most likely to present deletions, replications and/or unique arrangements compared with Archetype or Prototype. The type IIS Archetype ${ }^{\mathrm{CY}} \mathrm{NCRR}$ sequence contains a single copy of all NCRR sequence sections observed in other JCV variants. ${ }^{5}$ Because the $v$ of Archetype ${ }^{C Y}$ is nine base pairs shorter than the $v$ of Prototype ${ }^{\text {Mad-I }}$, the nucleotide numbering for all base pairs, excluding I through 36 , has been modified accordingly. The ' $A$ ' identifies a point mutation from ' $G$ ' that is always found in type IS and IR JCV NCCR sequences.

\section{Acknowledgements}

JC virus diagnostics were initially performed in Dusseldorf and Munich (Germany) and subsequently confirmed at the National Institutes of Health (NIH) (Dr Gene Major, Bethesda, USA). We thank Dr Christina McTamany for language-editing the manuscript. The authors thank Prof Kieseier and Dr Warnke (Dusseldorf) for kindly providing pre Natalizumab serum samples for anti-JCV antibody testing.

\section{Conflict of interest}

R. H. is supported by the Deutsche Forschungsgemeinschaft (SFB / TR 128) and by the Deutsche Forschungsgemeinschaft, Bundesministerium für Bildung und Forschung (BMBF-KKNMS) and has received personal compensations from Bayer Schering Pharmacy, Teva, Merck-Serono, Biogen Idec and Novartis. T.K. has received travel expenses and personal compensations from Bayer Schering Pharmacy, Teva, Sanofi-Aventis, Novartis, Merck-Serono and Biogen Idec as well as grant support from Bayer-Schering AG. J.H. received travel expenses and personal compensations from Merck-Serono, Teva, Bayer Schering, Novartis and Biogen Idec. C.R, P.J and E.M have no conflicts nor anything to report. A.B. has received travel expenses and personal compensations from Biogen Idec, Bayer, Teva and Novartis and grant support from Bayer. B.H. is supported by the Deutsche Forschungsgemeinschaft, Bundesministerium für Bildung und Forschung (BMBF-KKNMS), and Hertie Foundation, has received personal compensations from Hoffmann la Roche, Merck-Serono, Biogen Idec, Novartis and Bayer. He serves as a consultant for Gerson
Lehrman Group, and has received grant support from Novartis, Biogen Idec, Merck- Serono, Bayer, Metanomics and Protagen AG. A.J. has received travel expenses from Merck-Serono, Bayer, Teva, GlaxoSmithKline, Allergan and Abbott. H.K. has received travel expenses from Merck-Serono. K.L. reports no disclosures.

\section{Funding}

This research received no specific grant from any funding agency in the public, commercial, or not-for-profit sectors.

\section{References}

1. Kappos L, Bates D, Edan G, et al. Natalizumab treatment for multiple sclerosis: Updated recommendations for patient selection and monitoring. Lancet Neurol 2011; 10: 745-758.

2. Kuhle J, Gosert R, Buhler R, et al. Management and outcome of CSF-JC virus PCR-negative PML in a natalizumab-treated patient with MS. Neurology 2011; 77: 2010-2016.

3. Jensen PN and Major EO. A classification scheme for human polyomavirus JCV variants based on the nucleotide sequence of the noncoding regulatory region. $J$ Neurovirol 2001; 7: 280-287.

4. Frisque RJ, Bream GL and Cannella MT. Human polyomavirus JC virus genome. J Virol 1984; 51: 458-469.

5. Yogo Y, Iida T, Taguchi F, et al. Typing of human polyomavirus JC virus on the basis of restriction fragment length polymorphisms. J Clin Microbiol 1991; 29: 2130-2138. 